International Journal of Wireless \& Mobile Networks (IJWMN) Vol. 6, No. 2, April 2014

\title{
New Strategy to optimize the Performance of SPRAY AND WAIt ROUTING PROTOCOL
}

\author{
Mohamed Ababou ${ }^{1}$, Rachid Elkouch ${ }^{2}$, Mostafa Bellafkih ${ }^{3}$ and Nabil Ababou ${ }^{4}$ \\ ${ }^{1}$ National Institute of Posts and Telecommunications - INPT, Rabat, Morocco \\ University of sciences Mohamed 1 -FSO, Oujda, Maroc
}

\begin{abstract}
Delay Tolerant Networks have been (DTN) have been developed to support the irregular connectivity often separate networks. The main routing problem in this type of network is embarrassed by time that is extremely long, since connections are intermittent and opportunistic. Routing protocols must take into account the maximum constraint encountered in this type of environment, use effective strategies regarding the choice of relay nodes and buffer management nodes to improve the delivery of messages and the time of their delivery. This article proposes a new strategy that optimizes the routing Spray and wait. The proposed method uses the information contained in the messages delivered mostly paths traversed by the messages before arriving at their destination and the time when nodes have receive these messages. Simulation results show that the proposed strategy can increase the probability of delivery and minimizing overhead unlike FIFO technology used with the default routing ' sprat and wait'
\end{abstract}

\section{KEYWORDS}

DTN, Store and forward, networks, forwarding strategies, buffer management, routing, epidemic, spray and wait, Queue modes, FIFO.

\section{INTRODUCTION}

The objective of DTN networks [1] is to allow subnet that they know the weak communications in environments where the connection is intermittent, to routes messages between nodes using the principle of store and forward. In this type of network, nodes can forward messages to others nodes when they enter their transmission range. Due to the mobility of nodes, there is no guarantee to find at path between source and destination. To overcome this problem, DTN network uses the mechanism of messages replication to increase the chance that one of the message copies reaches its destination and uses information flowing through the network to choose the . The epidemic routing [2] and spray Wait [3] are among several which sent messages in DTN network using the replication mechanism and PROPHET [4] that uses the network information to choose the relay node .To evaluate the performance of these routing protocols in the absence of real traces, several simulator was developed as the network simulator (NS) and the opportunistic network environment simulator (the one) [5]. The evaluation is based on several metrics, such as the delivery probability, overhead ratio, the delivery rate and the number of hops that have carried messages.

This article proposes a strategy called 'Supp - copiesleft -Tran' to improve the performance of spray and wait routing protocol in terms of delivery probability, overhead ratio and Hop count average. The remaining paper is prearranged as follows .Section 2 elaborates existing forwarding

DOI : 10.5121/ijwmn.2014.6205 
International Journal of Wireless \& Mobile Networks (IJWMN) Vol. 6, No. 2, April 2014

strategies. Section 3 is about routing protocol in DTN networks, Section 4 About mobility models used, Section 5 develops performance metrics, Section 6 the new forwarding strategy, Simulation and results simulates in section 7 by a conclusion at section 8 .

\section{ForWARDing STRATEgIES}

\subsection{GRTR}

In this strategy the encounters nodes try to calculate the delivery predictability between them and the destination of message $\mathrm{P}(\mathrm{A}, \mathrm{D})$ and $\mathrm{P}(\mathrm{B}, \mathrm{D})$ which denotes respectively the delivery predictability [6] that a node A and B have for a destination message. the nodes that has the greater delivery predictability carries a message

\subsection{MOFO}

This strategy use the number of how many times that message has been sent to others hops and order it according to a descending order, the more the number of the sent message is low, the more the message has a chance of being transmitted [7]. If the buffer is full the strategy deletes messages that have been sent many times. Messages that were sent several times are not sent and the ones that have not been sent or transmitted many times are sent because this increases their chances of reaching their destination.

\subsection{Location Based Routing}

This strategy uses the coordinates of nodes such as GPS coordinates, to determine the distance between its locations and hops location, and then the message will be forwarded to the one that is closer in the coordinate space than the current custodian to destination.

\subsection{First in First out (FIFO)}

In FIFO queue mode all messages are arranged according to arrival time and the message which has oldest arrival time will be transmitted first [8].

\section{PROTOCOL UNDER OBSERVATION}

\subsection{Spray and Wait Routing Protocol}

Spray and Wait [3] routing protocol has been proposed to reduce the total number of copies sent across the network as is the case of epidemic routing. It uses the principle of epidemic routing but with a different strategy, in the spray phase, the nodes sent L copies to neighboring nodes and in the wait phase each relay nodes carrying the message until they meet the destination. in binary spray and wait version ,each node sends half number of message copies to each node encountered in its path until that they have only one copies where they will wait until they meet destination of the messages.

\section{ABOUT MOBILITY MODELS USED}

\subsection{SPMBM}

SPMBM (Shortest Path Map Based Movement Model) [4] is a more realistic model it manages the movement of nodes in the simulation map scenario. It will offer destination coordinates, 
International Journal of Wireless \& Mobile Networks (IJWMN) Vol. 6, No. 2, April 2014

speeds, wait times, and uses Dijkstra's algorithm to find the shortest path to the destination. It places the nodes in random places but selects a certain destination in the map for all nodes.

\subsection{Map Route Movement}

Map Route Movement MBM [4], is based on a map of a given region, where nodes can randomly choose their future destination, provided they follow the predefined routes on the map.

\section{THE PERFOMANCE METRICES MEASURED}

As it was stated in many researches, in order to compare routing strategies, some parameters need to be defined to evaluate their performance. The number of the selected metrics depends on several factors. it will be in relative terms.

\subsection{Overhead ratio}

Overhead ratio [4] can be defined as the subtraction of delivered (BD) from the bundle carried (BC) over bundles carried ((BC-BD)/BC).The overhead ratio reflects how many unnecessary messages are relayed to deliver one message. It reflects transmission cost in a network. The more the value of overhead is low the more the strategy used is efficient; this leads to a minimization of consumption of the network resources.

\subsection{Delivery probability}

This metric can be defined as ratio of the messages delivered over messages relayed._The higher values of probability mean that the performance of the algorithm is better.

\subsection{Hop count average}

It is the mean of the number of hops which participate to relayed message from its source to its destination successfully; higher values mean that the message has consumed many network resources before reaching its destination

\section{THE STRATEGY SUPP-COPIESLEFT-TRAN}

All nodes are mobile with a low density. There is no connection from end to end, and the movement of nodes affects the delivery of the message. When two nodes meet each other, they exchange the messages that they carry.

\subsection{Cleaning the buffer}

\subsubsection{Phase gathering information.}

When two nodes A and B meet, they exchange their two lists namely the list of neighbors and the list of unnecessary messages: 
International Journal of Wireless \& Mobile Networks (IJWMN) Vol. 6, No. 2, April 2014

\section{- List of neighbors}

$\mathrm{VA}=\left\{\mathrm{VA}_{\mathrm{i}}=\mathrm{Ai}, \mathrm{i}=1, \ldots, \mathrm{n}\right\}$ with $\mathrm{n}$ : number of neighbors of $\mathrm{A}$.

$\mathrm{VB}=\left\{\mathrm{VB}_{\mathrm{i}}=\mathrm{B}_{\mathrm{i}}, \mathrm{i}=1, \ldots, \mathrm{m}\right\}$ with $\mathrm{m}$ : number of neighbors of $\mathrm{B}$.

$\mathrm{VA}_{\mathrm{i}}$ and $\mathrm{VB}_{\mathrm{i}}$ are neighbors respectively of $\mathrm{A}$ and $\mathrm{B}$.

\section{- List of unnecessary messages.}

ListeMAN: List of messages that a node must not accept, it contains messages already arrived at their destinations.

ListeMAN (VAi): The list of messages that the neighbor $\left(\mathrm{A}_{\mathrm{i}}\right)$ of Node $(\mathrm{A})$ shall not accept.

ListeMAN $(A)=\left\{\right.$ ListeMAN $\left.\left(\mathrm{VA}_{\mathrm{i}}\right), \mathrm{i}=1, \ldots, \mathrm{n}\right\}$ where $\mathrm{n}:$ the number of neighbors of $\mathrm{A}$.

ListeMAN $(B)=\{$ ListeMAN $(V B i), i=1 \ldots \mathrm{m}\}$ with $\mathrm{m}$ : the number of neighbors of $\mathrm{B}$.

\section{-Creation of ListeMAN (AB)}

Creation of a new list that contains the messages to remove, by the union of the two previous lists - that is to say those that emanate from multiple nodes - in order to increase the removal rate of unnecessary messages circulating in the network even if a copy has already arrived successfully to its final destination, so their elimination minimizes the consumption of network resources due to the continuity of their dispersion in the network.

ListeMAN $(\mathrm{AB})=\left\{\right.$ ListeMAN $\left.\left(\mathrm{VA}_{\mathrm{i}}\right)\right\} \cup\left\{\operatorname{ListeMAN}\left(\mathrm{VB}_{\mathrm{i}}\right)\right\}$

\section{- Creating a list of common neighbours}

Each node compares the list of neighbours it receives with its own list to find the common neighbor nodes.

$\mathrm{VA}=\left\{\mathrm{VA}_{\mathrm{i}}=\mathrm{A}_{\mathrm{i}}, \mathrm{i}=1 \ldots \mathrm{n}\right\}$ where $\mathrm{n}$ is the number of neighbors of $\mathrm{A}$.

$\mathrm{VB}=\{\mathrm{VBi}=\mathrm{Bi}, \mathrm{i}=1 \ldots \mathrm{m}\}$ with $\mathrm{m}$ : number of neighbors of $\mathrm{B}$.

$\mathrm{VA} \cap \mathrm{VB}=\left\{\mathrm{N}_{\mathrm{i}}, 1 \ldots \mathrm{n}\right\}$

$\mathrm{n}$ : number of neighbors shared between A and B.

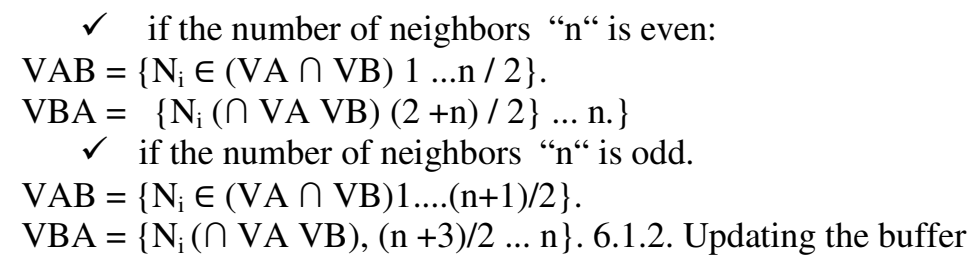

After creating the list of messages that nodes should not accept in their future contacts and list of common neighbors (VA $\cap \mathrm{VB}$ ), the two nodes $\mathrm{A}$ and $\mathrm{B}$ function like the following: - They clean their buffers by eliminating unnecessary messages in the list ListeMAN (AB). - Node A send a list ListeMAN (AB) to its neighbors except those in the list VBA that will be supported by node $B$. - Node B send a list ListeMAN ( $\mathrm{AB}$ ) to its neighbors except those in the list VAB that will be dealt by node A. This implies that the neighbors of two nodes clean their buffers by eliminating unnecessary messages in the ListeMAN list 
International Journal of Wireless \& Mobile Networks (IJWMN) Vol. 6, No. 2, April 2014

With this technique it was possible to increase the rate of messages to eliminate to release buffers of neighboring nodes and increase the probability that a Message previously received by its destination stops circulating in the network.

\subsubsection{Transmission of messages}

\subsubsection{Form of messages}

Table 1 Information Contained In Message.

\begin{tabular}{|l|l|l|l|l|}
\hline Message & $\mathrm{S}$ & $\mathrm{D}$ & $\left(\mathrm{hop}, \mathrm{t}_{\mathrm{i}}\right)=\left(\mathrm{N}_{1}, \mathrm{t}_{1}\right) \ldots\left(\mathrm{N}_{\mathrm{n}}, \mathrm{t}_{\mathrm{n}}\right)$ & Copies left \\
\hline
\end{tabular}

TABLE 2 Information Contained In Acknowledgement Message.

\begin{tabular}{|l|l|l|l|l|}
\hline Ack & $\mathrm{D}$ & $\mathrm{S}$ & $\left(\mathrm{K}_{\mathrm{i}}, \mathrm{t}_{\mathrm{i}}\right)=\left(\mathrm{k}_{1}, \mathrm{t}_{1}\right) \ldots\left(\mathrm{K}_{\mathrm{n}}, \mathrm{t}_{\mathrm{n}}\right)$ & $\left(\mathrm{S}, \mathrm{t}_{0}\right)\left\{\left(\mathrm{N}_{\mathrm{i}}, \mathrm{t}_{\mathrm{i}}\right)\right\}\left(\mathrm{D}, \mathrm{t}_{\mathrm{n}+1}\right)$ \\
\hline
\end{tabular}

S: Source D: destination,(hop, $t_{i}$ ) :hop involved in transport of the message. $\mathrm{t}_{\mathrm{i}}$ : the time when the message has been transmitted to $\mathrm{Ni}$, Size: the size of the message.Copies left: the number of copies remaining to be transmitted.

(Ki, ti) hop who participated in the transport of acknowledgement messages.

$\left\{\left(\mathrm{S}, \mathrm{t}_{0}\right)\left\{\left(\mathrm{N}_{\mathrm{i}}, \mathrm{t}_{\mathrm{i}}\right)\right\}\left(\mathrm{D}, \mathrm{t}_{\mathrm{n}+1}\right)\right\}$ the path that the message took to go from the source to the destination.

\subsubsection{The Priority List Creation .}

Each node classifies messages in its buffer; example node A searches for messages that have as destination node $\mathrm{B}$, it range them in descending order based on their injection in the network and in ascending order based on their size and it is at this level that starts transmission.

\section{- Method for creating the priority list:}

Node A consults at the buffer messages before deciding:

- If $B$ is the source: it ignores the message.

- If $\mathrm{B}$ is the destination: it added to the priority list.

- If B belongs to the hop list who participated in the transport of the message to the moment of contact: it ignores the message.

\subsubsection{Transmission of Priority List}

Once the priority list is created the node classifies the messages in its buffers ordering to the number of copies left before forwarding the messages in this list to node B.

\subsubsection{Transmission or Non-Transmission of Other Messages.}

In the case where the node $\mathrm{B}$ is not in the set of hops $\{(\mathrm{S}, \mathrm{D},\{(\mathrm{Ni}, \mathrm{Ti})\}\}$ that is to say, it is neither a source nor a destination, nor the one of the nodes that participated in the transfer of messages, 
node $\mathrm{A}$ uses the information contained in the list of paths supplied by the set of paths extracted from the list ListeMAN $(\mathrm{AB})$ :

$\{(\mathrm{Sj}, \mathrm{t} 0)\{(\mathrm{Ni}, \mathrm{ti})\}(\mathrm{Dj}, \mathrm{tn}+1)\}$ : list of paths of messages successfully transmitted to their destination. $\{(\mathrm{Dj}, \quad$ tn +1$) \quad\{(\mathrm{Ki}, \quad \mathrm{ti})\} \quad(\mathrm{Nj}, \quad \mathrm{tn})\}:$ list of acknowledgments paths. Node A, creates a list of pairs of nodes $(\mathrm{Ni}, \mathrm{D})$ called:

Listech $(\mathrm{D}, \mathrm{Ni})=\{$ vector(frequency, hops average number $)\}$ which means that it extracts from all messages paths in list ListeMAN (AB), the number of times(frequency) when the two nodes are involved with delivering messages to their success destination and the average number of hops that separate in each path, then classifies messages in ascending order based on the number of occurrence of nodes $\mathrm{Ni}$ and D (frequency) and in descending order of hop number between them, thereafter, it selects from its neighbors which must carry the message. The nodes $\mathrm{Ni}$ and $\mathrm{D}$ are very close if the number of hops between them is minimal, in this case there is a strong chance that the message reaches its destination without any problems.

Once the secondary list is created the node classifies the messages in its buffers ordering to the number of copies left before forwarding the messages in this list to appropriate node.

\section{EXAMPLE}

Assume A, B ,C,E,F and D are five intermittently connected mobile nodes and $\{\mathrm{B}, \mathrm{C}, \mathrm{F}, \mathrm{E}\}$ are neighbors of A see Figure 1 and Table 3represents the messages buffered at node A and node B, previous to the transmission and Table 4 shows the lists of messages delivered successfully to their destination by each nodes. It is assumed that each message transmission time is 1 second while total DTN transmission time is 4 seconds.

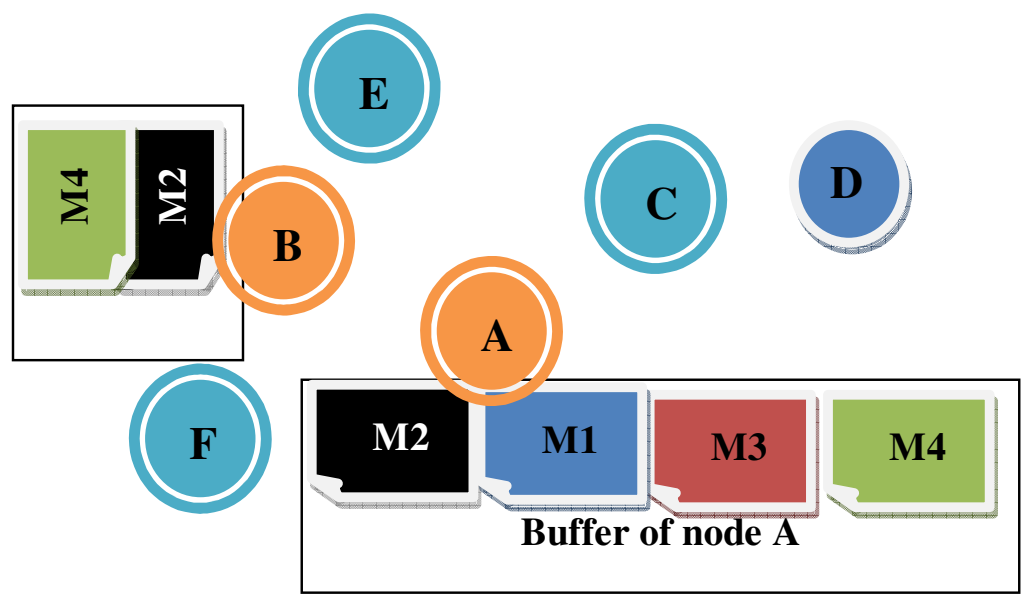

Figure 1 snapshot of encounters nodes before communication between A and B

\begin{tabular}{|c|c|c|c|}
\hline ListeMAN(A) & ListeMAN(B) & ListeMAN(C) & ListeMAN(E) \\
\hline M6 & M1 & M3 & M4 \\
\hline M17 & M11 & M12 & \\
\hline M81 & $\mathrm{M} 12$ & M13 & \\
\hline M19 & M13 & & \\
\hline $\mathrm{M} 12$ & & & \\
\hline
\end{tabular}


International Journal of Wireless \& Mobile Networks (IJWMN) Vol. 6, No. 2, April 2014

\begin{tabular}{|l|} 
M11 \\
\hline M12 \\
\hline M13 \\
\hline
\end{tabular}

Figure 3 List of messages already deleted by node A, B ,C and E

\begin{tabular}{|c|c|c|c|c|c|c|c|}
\hline \multicolumn{4}{|c|}{ Buffer of Node A } & \multicolumn{4}{c|}{ Buffer of Node B } \\
\hline Msg & Destination & Copies Left & Arrival Time & Msg & Destination & Copies Left & Arrival Time \\
\hline M1 & D & 5 & 150 & M17 & D & 4 & 224 \\
\hline M2 & D & 5 & 123 & M2 & D & 2 & 224 \\
\hline M3 & D & 4 & 60 & M5 & D & 4 & 204 \\
\hline M4 & D & 4 & 15 & M6 & D & 5 & 99 \\
\hline
\end{tabular}

Figure 4 snapshot of messages buffered in node A and B before communication

\section{Case 01: Forwarding Sequence with FIFO strategy}

Table 4 represents the array of messages at node A and B before the transmission. With FIFO strategy, node A transmits the messages:

Node A forward the message:

$\{\mathrm{M} 3, \mathrm{M} 4\}$ to node B except $\{\mathrm{M} 1, \mathrm{M} 2\}$, which was already transported them $\{\mathrm{M} 1, \mathrm{M} 2, \mathrm{M} 4\}$ to node $\mathrm{C}$ except M3 and $\{\mathrm{M} 1, \mathrm{M} 2, \mathrm{M} 3\}$ to node E except M4

Figure 2 shows the messages buffered at neighbor's node A after transmission. The result shows that the messages M1, M3 and M4 continue to circulate in the network, even if one of its copies was delivered to its final destination. This causes an unnecessary consumption of network resources. 


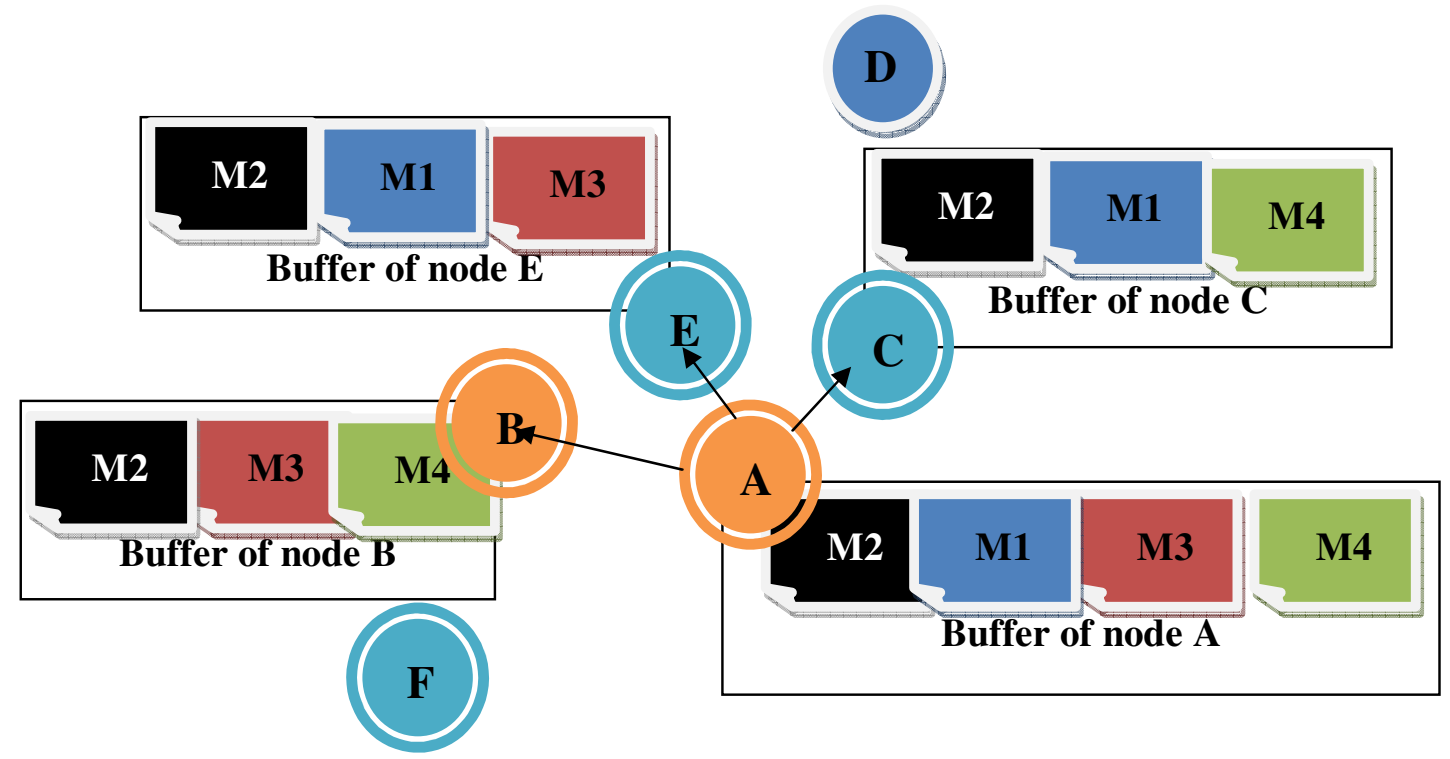

Figure2. Messages buffered in neighbors' node A after transmission with FIFO strategy

\section{Case 02: Forwarding sequence with "Supp-copiesleft-Tran" strategy}

Table 5 depicts the organization of messages at node A by Supp-copiesleft-Tran previous to transmission.

With sup-copiesleft-tran strategy, node A follows the following steps before messages forwarding:

Step 1: Node A exchanges first, the list 'LISTMANT' [Table 3], with its neighbors and thereafter, forms the global list that will contain the messages' identifiers of already delivered messages.

\begin{tabular}{|l|l|l|l|l|l|l|l|l|}
\hline \multirow{2}{*}{ ListeMAN(A) } & M6 & M17 & M81 & M19 & M12 & M11 & M12 & M13 \\
\cline { 2 - 9 } & M1 & M11 & M12 & M13 & M3 & M12 & M13 & M4 \\
\hline
\end{tabular}

Table 5. List 'LISTMANT' created by node A in Tran phase

Step 2: Node A updates its buffer and sends the list 'LISTMANT' [Table 5] formed to its neighbors for update their buffer.

Step 3: The node A sends messages that remain in the buffer based on the list of paths 'LISTECH' [Table 6] which means, that after it updated its buffer, node A removes messages that are successfully delivered, that is to say, the messages M1,M3 and M4, it still has only M2 which will forward it to node C, the more likely to meet node D. Figure 3 shows that the technique avoids the transmission of unnecessary messages if one of its copies arrived at its destination. 
International Journal of Wireless \& Mobile Networks (IJWMN) Vol. 6, No. 2, April 2014

Table 6. LISTECH(D,Ni) of node a Created By "Supp-copiesleft-Tran" in Section 6.

\begin{tabular}{|c|c|c|}
\hline pair of nodes & frequency & $\begin{array}{c}\text { Average } \\
\text { hops }\end{array}$ \\
\hline D:C & 11,7 & 0 \\
\hline D:B & 5,87 & 3 \\
\hline D:H & 3 & 3 \\
\hline D:F & 3 & 5 \\
\hline D:E & 2 & 7 \\
\hline D:A & 1 & 1 \\
\hline
\end{tabular}

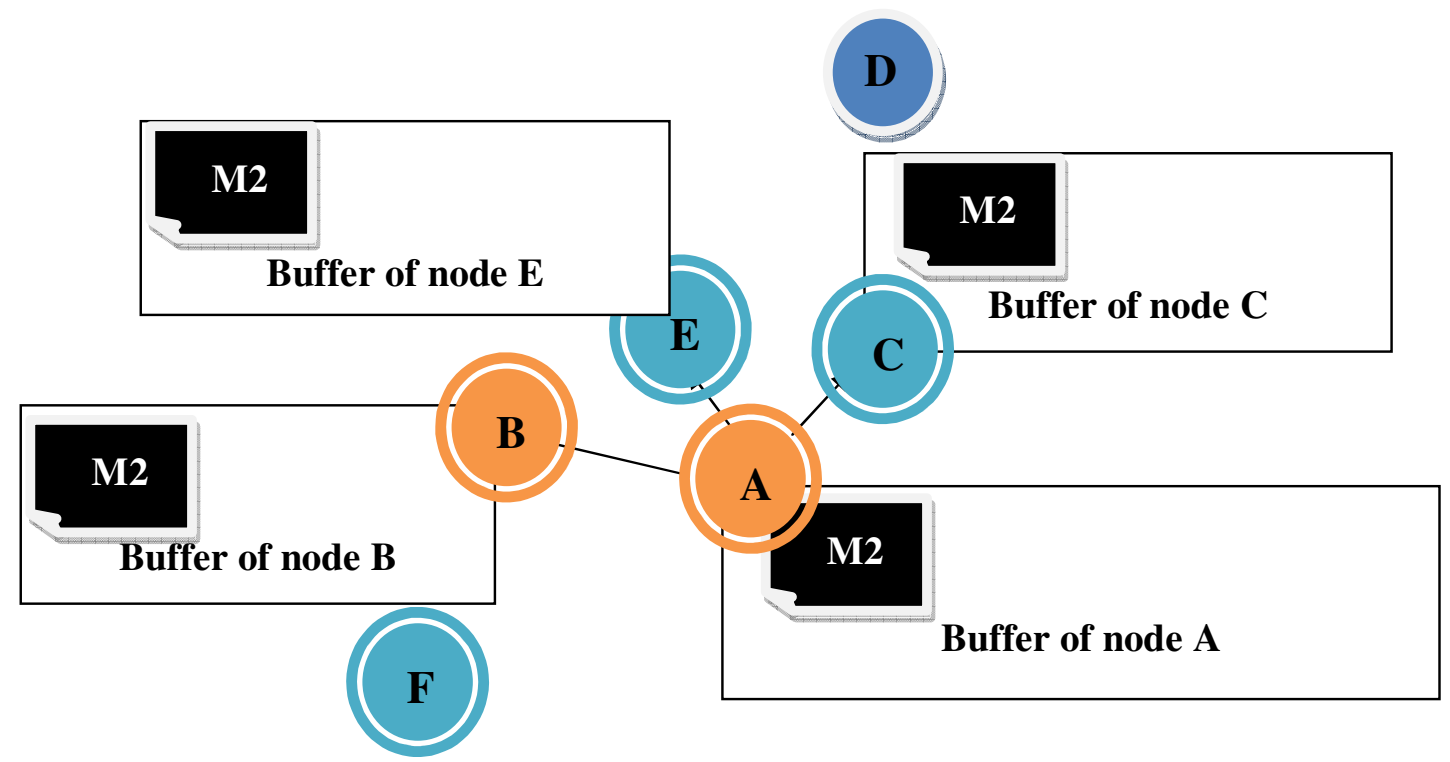

Figure 3 Messages buffered in neighbors' node A after transmission with 'SUP-COPIESLEFT-TRAN' strategy

\section{SIMULATION AND RESULTS}

\subsection{Simulation Environments}

This section presents the results of the comparison between the forwarding strategies FIFO and Sup-Tran presented in Section 6, regarding their effectiveness under spray and wait routing protocols. All simulations are done with the ONE (The Opportunistic Network Environment Simulator) [1] written in Java to evaluate the performance of the two methods. The ONE was created by Helsinki University and provided the map of the Helsinki area.

In the simulation, there are two different nodes that may generate and receive a message. One is a pedestrian and the other is a vehicle. The number of two different nodes is 40 and 6 respectively for pedestrian and vehicle. These two types of nodes are moving with different mobility along the map of Helsinki area.

For the pedestrian, the walking speed (i.e., $0.5 \mathrm{~m} / \mathrm{s} \sim 1.5 \mathrm{~m} / \mathrm{s}$ ) is applied. The moderate speed 
International Journal of Wireless \& Mobile Networks (IJWMN) Vol. 6, No. 2, April 2014

(i.e., $6 \mathrm{~m} / \mathrm{s} \sim 12 \mathrm{~m} / \mathrm{s}$ ) is applied to the vehicle. The details of the simulation parameters are shown in Table 7.

Table 7. Simulation parameters

\begin{tabular}{|l|l|}
\hline Simulation Parameters & Simulation values \\
\hline Number of nodes & 200 nodes (P: 100, V: 100) \\
\hline Simulation time & $\begin{array}{l}\text { 10k-80K for simulation w.r.t time } \\
10 \mathrm{~K} \text { for simulation w.r.t } \\
\text { Transmission range }\end{array}$ \\
\hline Node movement & $\begin{array}{l}\text { Shortest path map based movement, } \\
\text { Map Route Movement }\end{array}$ \\
\hline Transmission range & $\begin{array}{l}10 \mathrm{~m} \text { for simulation w.r.t time } \\
10 \mathrm{~m}-120 \mathrm{~m} \text { for simulation w.r.t } \\
\text { Transmission range }\end{array}$ \\
\hline Packet transmission speed & $250 \mathrm{Kbyte} / \mathrm{s}$ \\
\hline Buffer size & $10 \mathrm{M}, 50 \mathrm{M}$ \\
\hline
\end{tabular}

\subsection{Performance Analysis}

To evaluate the proposed method compared with FIFO under spray and wait routing protocol, the simulation has to consider performance metrics such as delivery rate, communication overhead, and number of hops. It is evident that the higher delivery rate means better performance on successful data delivery. However, the effort to get the higher delivery rate, a routing protocol has to send the more data into the networks, these additional data packets may result in communication overhead. Therefore, both delivery rate and communication overhead are analyzed simultaneously.

Finally, average number of hops is compared to demonstrate consumption of resources. Clearly, DTNs accept a tolerable delay for message delivery. On the other hand, some applications do not accept higher number of hops to deliver messages from the source to the destination.

\subsection{Simulation Results}

All the results got from the simulation are shown below in form of graph representation and observations are discussed.

\subsubsection{By Varying Time Intervals}

\begin{tabular}{|l|c|c|l|c|c|}
\hline & \multicolumn{5}{|c|}{ Spray and wait } \\
\hline strategy & FIFO & $\begin{array}{c}\text { SUP- } \\
\text { copiesleft-tran }\end{array}$ & \multicolumn{1}{|c|}{ strategy } & FIFO & $\begin{array}{c}\text { SUP- } \\
\text { copiesleft-tran }\end{array}$ \\
\hline created & 336 & 336 & delivered & 120 & 133 \\
\hline started & 6700 & 6647 & Delivery probability & 0.3571 & 0.3958 \\
\hline relayed & 2617 & 2561 & Overhead ratio & 20.8083 & 18.2556 \\
\hline aborted & 4081 & 4084 & Latency average & 2182.3867 & 2403.303 \\
\hline dropped & 1993 & 1286 & Hop count average & 2.8 & 2111.8 \\
\hline removed & 0 & 759 & Hop count average & 2.8 & 2111.8 \\
\hline
\end{tabular}

Table 8. Example of result of simulation with $\quad 10000$ seconds 
Figure 4 shows that increasing the simulation time from $10 \mathrm{~K}$ to $80 \mathrm{~K}$ increases the probability of deliverance for both method, which can be explained as more nodes are traveling for a long time, the more they encounter another nodes so they may exchange more messages, but for each time interval, the results show that the strategy Supp-copiesleft-Tran has better performance than the FIFO strategy in terms of messages that can be delivered to their destination.

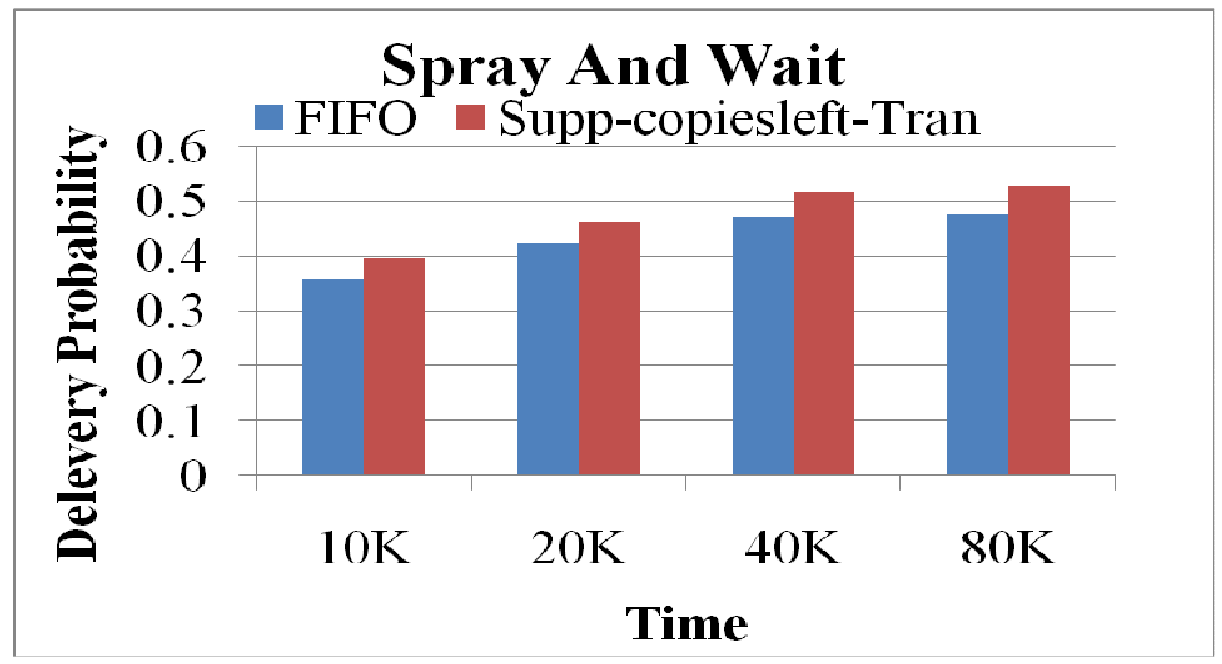

Figure 4. Delivery Probability w.r.t

Figure 5 shows the results of the evaluation in terms of the overhead ratio of FIFO and Suppcopiesleft-Tran by varying the time interval from $10 \mathrm{~K}-80 \mathrm{~K}$ under spray and wait routing protocol. As shown, the overhead generated by the proposed method is lower than the FIFO strategy in all interval time, which means that with Supp-copiesleft-Tran strategy, the number of messages that continue traveling in the network without reaching their destination. Unlike, with FIFO strategy where this number is higher which means that the messages moving around the network for a long time which causes that the messages consume the network resource.

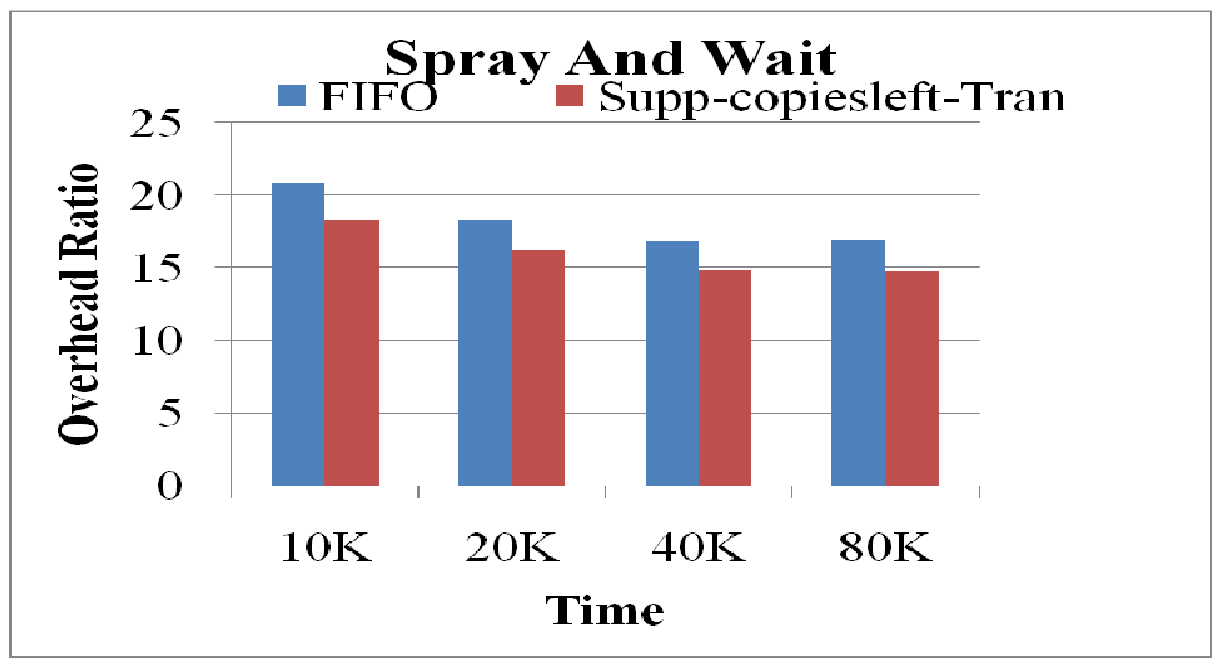

Figure 5. Overhead ratio w.r.t Time 
Figure 6 explains the average hops number of the proposed strategy compared to FIFO technique, as the result show, the proposed approach has a smaller pattern of the average hops number. It can see clearly that at various time intervals the hop-count average of "Supp-copiesleft-Tran" is lower than FIFO. The proposed method uses a number of hops slightly greater than the strategy FIFO which is evident because the Supp-copiesleft-Tran method tries to choose the best hops having a higher probability or greater chance to deliver messages, unlike the FIFO technique that transmits the messages based on their arrival time without checking if the relay nodes will encounter destination or not which causes that some nodes carry the messages even if it will never meet the destination, even if it has a small number of hops, it does not mean that is better because the delivery probability is low as its shown above in Figure5.

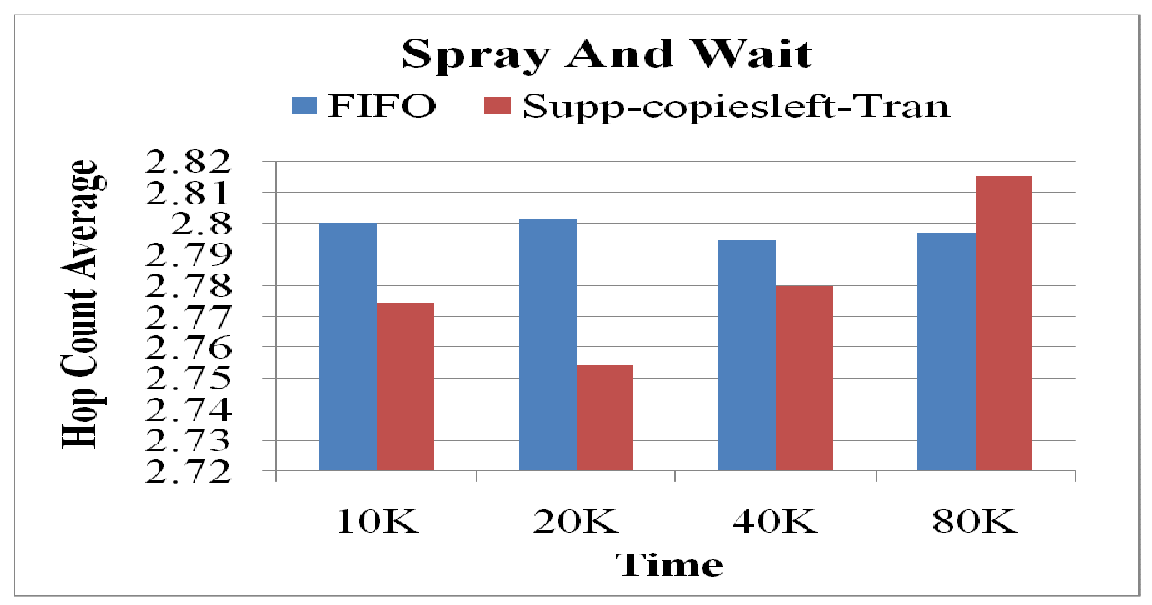

Figure . Hop count average w.r.t Time

\subsubsection{By Varying Transmission range}

Figure 7 compares FIFO and Supp-copiesleft-Tran strategy in terms of delivery probability by increasing the transmission range. The Supp-copiesleft-Tran strategy proves better delivery probability than FIFO, since increasing the transmission range increases the number of neighbors, which allows nodes using Supp-copiesleft-Tran to deliver the message as close as possible to their destination by choosing the most appropriate hop.

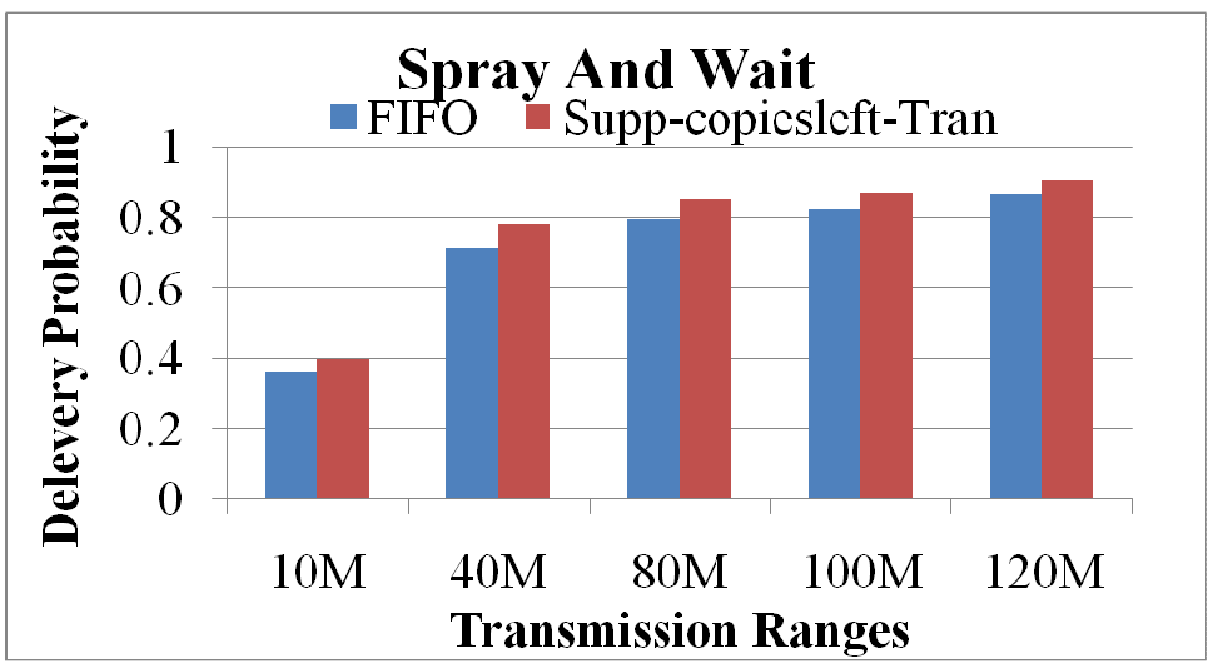

Figure 7. Delivery probability w.r.t Transmission range 
Figure 8 shows that in all transmission ranges the overhead of "Supp-copiesleft-Tran" is lower than FIFO strategies. This means that the strategy Supp-copiesleft-Tran is able to choose the best relay to transport messages to the right destination that is reflected in the low value of overhead.

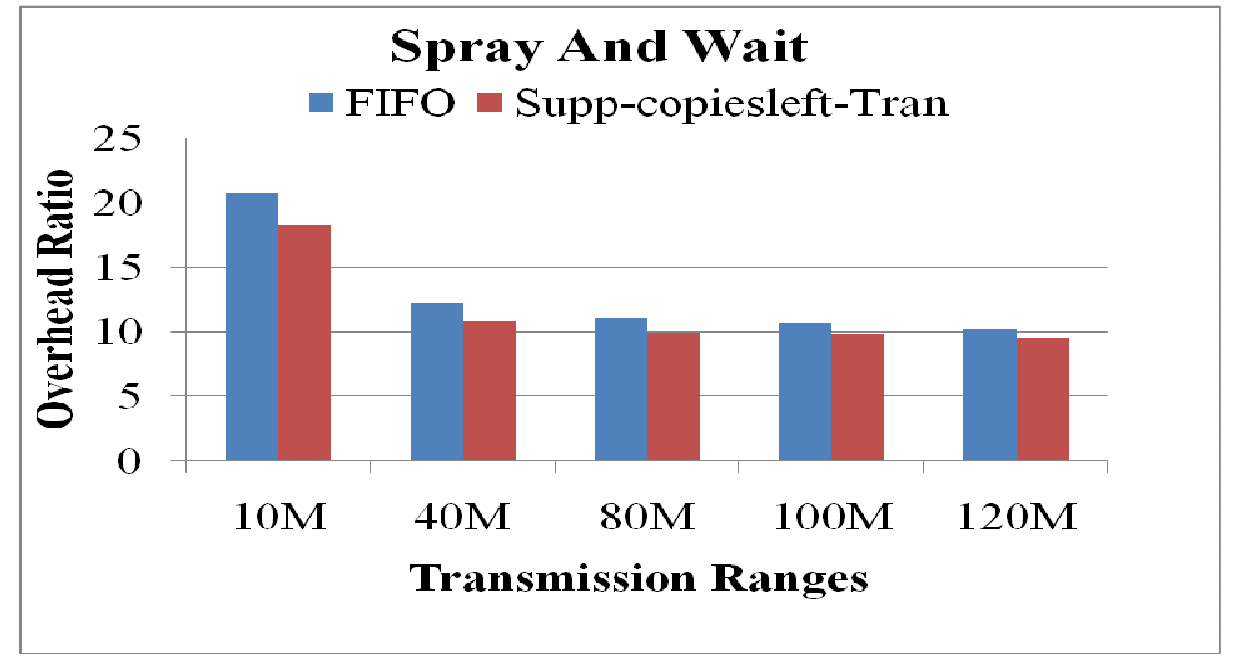

Figure 6. Overhead ration w.r.t Transmission range

Fig 7 presents the impact of transmission range on hop count. It shows that the hops count with FIFO strategy is lower than Supp-copiesleft-Tran. The sup-Tran, especially in the delivery probability and overhead ratio, is more efficient than FIFO.

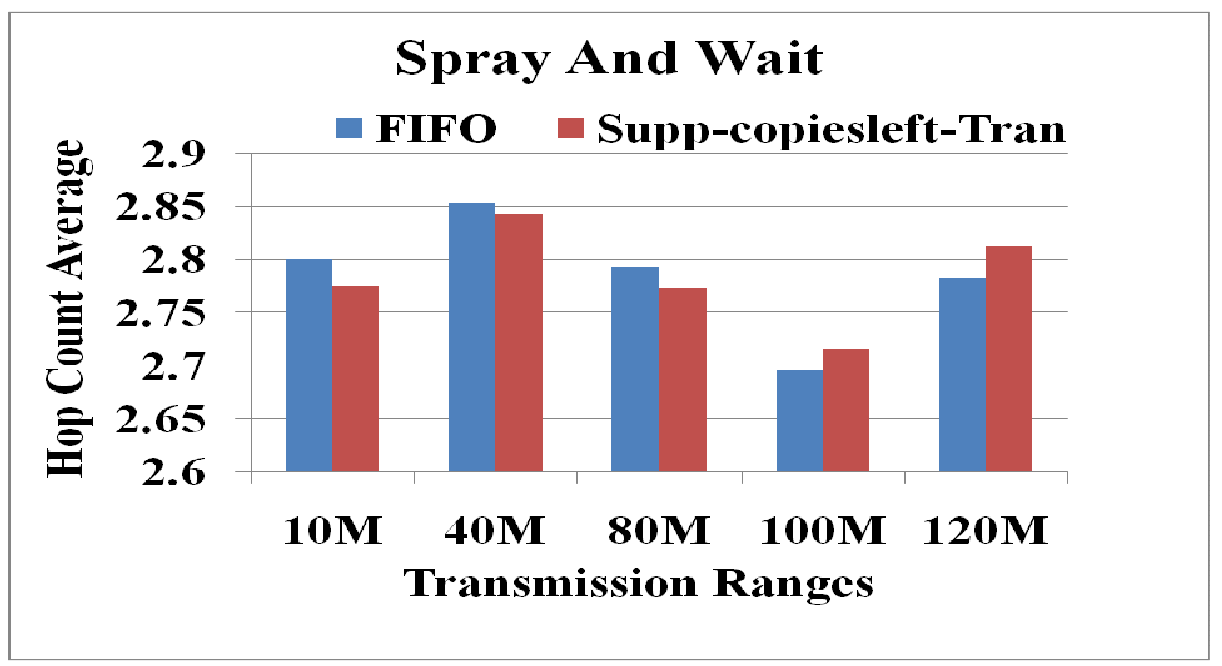

Figure 7. hop count Average w.r.t Transmission range.

\section{CONCLUSIONS}

This article proposes a strategy called " Supp-copiesleft -Tran ", which aims to optimize spray and wait routing protocol in delay tolerant networks, to use the network resources in an efficient manner contrary to the FIFO technique, as its name indicates, the proposed technique consists of three phases, the 'Supp' which means that the encountered nodes start to clean their buffer before deciding to transmit messages that are in their buffer. They exchange the list of messages that are 
International Journal of Wireless \& Mobile Networks (IJWMN) Vol. 6, No. 2, April 2014

successfully delivered to delete the remaining copies that still circulate in the network to release their buffers as well as those of their neighbors. The phase 'copies left' the nodes organize the messages in their buffer in order to the number of messages copies that remaining, the ones that have not been sent or transmitted many times which increase their chances of reaching their destination.The phase 'Tran' starts with the transmission of the messages selected in phase 'copies left' based on the list of neighbors and the list formed by the paths of messages delivered to calculate the frequency of meeting between destination and neighboring nodes of the two nodes that are in communication as well as the number of hops between them. During the phase "Supp", a proposed strategy optimizes routing sprat and wait by increasing the rate of the removed copies of messages that have been delivered to their destination. Indeed, the list formed by considering the list of the deleted messages of the encountered nodes and their neighbors will be forwarded to the neighbors to clean their buffer. During the phase 'copies lift' the technique give priority or chance to the message that not been sent or transmitted many times, During the " Tran" Phase, the proposed technique optimizes routing sprat and wait in terms of selecting relay nodes that will be carrying the messages. In fact, it uses the paths contained in the deleting messages' list to calculate the number of times the relay nodes have met the final destination of messages. This technique enables the reduction of the overhead and increases the delivery probability of messages compared to FIFO strategy.

\section{REFERENCES}

[1] V. Cerf and al. 2001. Interplanetary internet (IPN): Architectural definition.

[2] A. Vahdat and D. Becker, (2000) Epidemic routing for partially-connected ad hoc networks, Tech. Rep.

[3] T. Spyropoulos, K. Psounis, and C. S. Raghavendra, (2005). Spray and wait: Efficient routing in intermittently connected mobile networks," in Proc.ACM SIGCOMM Workshop on Delay Tolerant Networking (WDTN).2005

[4] Phearin Sok, Keecheon Kim, (2013) Distance-based PRoPHET Routing Protocol in Disruption Tolerant Network.

[5] Ari Keränen, Jörg Ott and Teemu Kärkkäinen (2009) The ONE Simulator for DTN Protocol Evaluation.

[6] A. Lindgren and A. Doria, (2012) Probabilistic Routing Protocol for Intermittently Connected Networks.

[7] Ashraf, Al-Fagih, Hossam and S.Hassanein; (2012) Routing Schemes for Delay-Tolerant Networks : An Applications Perspective Technical Report .

[8] J. Crowcroft, E. Yoneki, P. Hui, and T. Henderson,( 2008) Promoting tolerance for delay tolerant network research, SIGCOMM.

Authors

Mohamed ababou: $\mathrm{PhD}$ student at the doctoral studies center, National Institute of Posts and Telecommunications Rabat, Morocco.His research interests are computer science, DTN Network. Master Course in Informatics Telecommunications \&\& Multimedia at Faculty of Science, Mohammed V- University, Rabat, Morocco.

Rachid Elkouch :The INPT prepares and coach engineers in networks Telecoms, Computer sc ience and Multimedia. These engineers could be future companies responsible, in a world open to the dynamic of the networks and information systems, PHD in Maths/Telecommunications - National Institute of Posts and Telecommunications.

Nabil ababou: PhD student at the doctoral studies center, Faculty Mohamed I oujda , Morocco.His research interests are computer science, research in programming language. Master Course in Informatics at Faculty of Science Mohamed 1, Oujda, Morocco
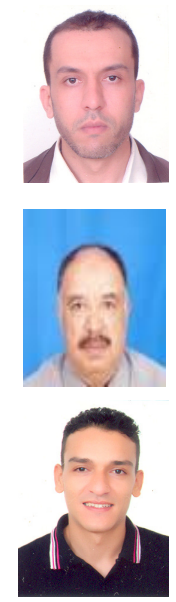